\title{
A POLG2 Homozygous Mutation in an Autosomal Recessive Epilepsy Family Without Ophthalmoplegia
}

\author{
Su Jeong Lee ${ }^{a}$ \\ Sumaira Kanwal \\ Da Hye Yoo ${ }^{\mathrm{a}}$ \\ Hye Ri Park \\ Byung-Ok Choi ${ }^{\mathrm{C}}$ \\ Ki Wha Chung ${ }^{a}$
}

aDepartment of Biological Sciences, Kongju National University, Gongju, Korea

'Department of Biosciences, COMSATS University Islamabad, Sahiwal, Pakistan 'Department of Neurology, Samsung Medical Center, Sungkyunkwan University School of Medicine, Seoul, Korea

Received January 11, 2019

Revised April 9,2019

Accepted April 9, 2019

\section{Correspondence}

Byung-Ok Choi, $\mathrm{MD}, \mathrm{PhD}$

Department of Neurology,

Samsung Medical Center,

Sungkyunkwan University

School of Medicine, 81 Irwon-ro, Gangnam-gu, Seoul 06351, Korea

Tel $+82-2-3410-1296$

Fax +82-2-3410-0052

E-mail bochoi@skku.edu

Ki Wha Chung, PhD

Department of Biological Sciences,

Kongju National University,

56 Gongjudaehak-ro, Gongju 32588

Korea

Tel +82-41-850-8506

Fax $+82-41-850-0957$

E-mail kwchung@kongju.ac.kr
Dear Editor,

Epilepsy is a chronic neurological disorder characterized by recurrent seizures with abnormal electrical activity. Epilepsy could be caused by both genetic and environmental factors. More than a thousand genes have been reported to be associated with epilepsy.

This study examined a Pakistani autosomal recessive epilepsy family (Fig. 1A). The patient was a 27-year-old male born after an uncomplicated pregnancy. He began to experience focal epileptic seizures with secondary generalization at the age of 23 years. Their frequency was irregular and so difficult to quantify, but seizures occurred roughly twice weekly. A combination therapy with valproate and carbamazepine as anti-epileptic drugs was applied, but this produced no response. Brain imaging performed at the same time showed normal findings. His deceased younger brother also had experienced intractable seizures that did not respond to valproate and carbamazepine. Neither patient had liver disease, ophthalmoplegia, ptosis, nystagmus, or myopathy, and their parents showed no symptoms of epilepsy or ophthalmoplegia.

Exome sequencing revealed seven rare functionally significant variants (allele frequencies $\leq 0.01$ ) from the $\sim 1,400$ reported epilepsy-related genes (Supplementary Table 1 in the online-only Data Supplement). Among them, a homozygous mutation (c.694G>A, p.G232S) in POLG2 (DNA polymerase gamma 2, accessory subunit) was only consistent with the recessive inheritance mode. This mutation was not observed in 300 controls. Although the POLG2 mutation was already registered in the dbSNP database (rs143660836), it was rarely reported in several public human genome databases, having allele frequencies of $\leq 0.0002$. Both of the unaffected parents of the patient were heterozygous for this mutation (Fig. 1B). The p.G232 site is well conserved among vertebrate species (Fig. 1C), and several in silico analyses have predicted a pathogenic effect for this mutation (Supplementary Table 2 in the online-only Data Supplement). Thus, we thought that the POLG2 mutation might act as likely pathogenic. The other six variants were not considered as the genetic cause due to their non-segregation with the affected family member. However, we could not completely rule out their pathogenicity, because mutations in some epilepsy genes show incomplete penetrance. ${ }^{2}$ They might have a role as association factors that influence the phenotypic variability.

Several POLG2 mutations have been reported in autosomal dominant progressive external ophthalmoplegia (adPEO, PEOA4: MIM 610131), with various additional symptoms of ataxia, tremor, myopathy, and seizure., Most POLG2 mutations are associated with long deletion(s) of mitochondrial DNA (mtDNA). Our patient and his mother showed a novel deletion in the mtDNA obtained from blood samples (Fig. 1D). The deletion occurred within m.8812-12296, with an exact size of 3,485 bp (Fig. 1E and F). Ten genes were completely or partially deleted by this deletion. A partial short inverted repeat sequence was present near the breakpoints (TTACACCAA/TTGGTGCAA). Once the mtDNA depletion had been determined, no significant difference in mtDNA content was observed between the

@ This is an Open Access article distributed under the terms of the Creative Commons Attribution Non-Commercial License (https://creativecommons.org/licenses/by-nc/4.0) which permits unrestricted non-commercial use, distribution, and reproduction in any medium, provided the original work is properly cited. 


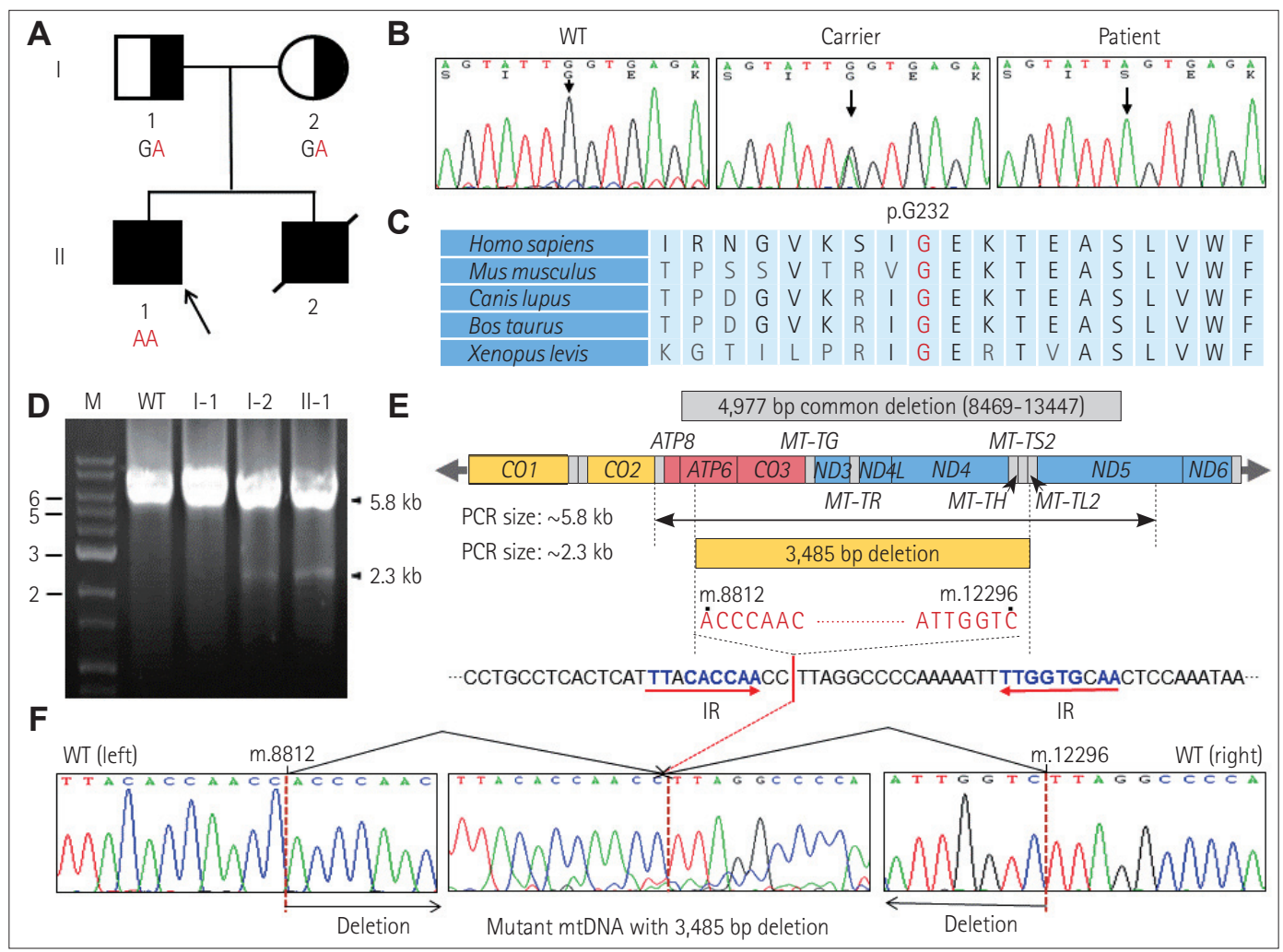

Fig. 1. A Pakistani epilepsy family carrying a POLG2 mutation and identification of the genetic causes. A: Pedigree of the autosomal recessive epilepsy family (family ID: EF-14). Genotypes of the POLG2 mutation are indicated below the symbols indicating the examined family members. Filled and semifilled symbols represent affected and carrier individuals, respectively. The proband is indicated by the arrow. B: Sequencing chromatograms showing the c.694G>A (p.G232S) mutation in POLG2. C: Conservation of the amino acids at the mutation site (p.G232S) among several vertebrates. D: Determination of a novel long mtDNA deletion. Each 5.8-kb and 2.3-kb band indicates the WT and long deletion of mtDNA (M, 1 kb ladder; WT mtDNA with no deletion). E: Schematic diagram of the 3,485 bp deletion (partial short IR sequences). F: Sequencing chromatograms showing the break points of 3,485 bp (left, upstream region of normal mtDNA; middle, mutant mtDNA with 3,485 bp deletion; right, downstream region of normal mtDNA). IR: inverted repeat, mtDNA: mitochondrial DNA, WT: wild type.

patient and gender- and age-matched controls (Supplementary Fig. 1 in the online-only Data Supplement).

POLG2 encodes a 55-kDa accessory subunit that increases the processivity of the catalytic subunit of DNA polymerase gamma encoded by POLG. More than 300 POLG mutations have been reported, but few POLG2 mutations have been reported as genetic causes. Most pathogenic mutations of POLG2 have been found to be the dominant type. ${ }^{3,4}$ There is a recent report of a patient with a homozygous POLG2 mutation having severe hepatic failure and mitochondrial depletion. ${ }^{5}$ The p.G232S described here is the second recessive mutation reported in POLG2.

Most mutations in POLG2 are implicated in PEO with the additional symptom of seizures. However, no PEO symptoms were observed in the present patient or both of his normal parents. Therefore, this might be the first report of a patient with a homozygous POLG2 mutation mainly associated with epilepsy. The findings of this study will help the molecular diagnosis of epilepsy by extending the range of clinical phe- notypes caused by POLG2 mutations.

\section{Supplementary Materials}

The online-only Data Supplement is available with this article at https://doi.org/10.3988/jcn.2019.15.3.418.

\section{Conflicts of Interest}

The authors have no potential conflicts of interest to disclose.

\section{Acknowledgements}

This work was supported by the National Research Foundation, Republic of Korea (2017R1A2A2A05001356, 2017R1A2B2004699, and 2018R1A4A 1024506).

\section{REFERENCES}

1. Wang J, Lin ZJ, Liu L, Xu HQ, Shi YW, Yi YH, et al. Epilepsy-associated genes. Seizure 2017;44:11-20.

2. Biervert C, Schroeder BC, Kubisch C, Berkovic SF, Propping P, Jentsch TJ, et al. A potassium channel mutation in neonatal human epilepsy. Science 1998;279:403-406.

3. Longley MJ, Clark S, Yu Wai Man C, Hudson G, Durham SE, Taylor 
RW, et al. Mutant POLG2 disrupts DNA polymerase gamma subunits and causes progressive external ophthalmoplegia. Am J Hum Genet 2006;78:1026-1034.

4. Young MJ, Longley MJ, Li FY, Kasiviswanathan R, Wong LJ, Copeland WC. Biochemical analysis of human POLG2 variants associated with mitochondrial disease. Hum Mol Genet 2011;20:3052-3066.

5. Varma H, Faust PL, Iglesias AD, Lagana SM, Wou K, Hirano M, et al. Whole exome sequencing identifies a homozygous POLG2 missense variant in an infant with fulminant hepatic failure and mitochondrial DNA depletion. Eur J Med Genet 2016;59:540-545. 